\title{
Preoperative and intraoperative extracorporeal membrane oxygenation adoption for long-term left ventricular assist device implantation
}

\author{
Bujar Maxhera ${ }^{1}$, Nihat Firat Sipahi ${ }^{1}$, Alexander Albert ${ }^{1}$, Udo Boeken ${ }^{1}$, Artur Lichtenberg ${ }^{1}$, Diyar Saeed ${ }^{1,2}$ \\ ${ }^{1}$ Department of Cardiovascular Surgery, University Hospital of Düsseldorf, Heinrich-Heine University, Düsseldorf, Germany; ${ }^{2}$ Department of \\ Cardiac Surgery, Leipzig Heart Center, University of Leipzig, Strümpellstrasse, Leipzig, Germany \\ Correspondence to: Diyar Saeed, MD, PhD. Professor of Surgery, Surgical Director of the MCS and Heart Transplantation Program, Department of \\ Cardiac Surgery, Leipzig Heart Center, University of Leipzig, Strümpellstrasse 39, 04289 Leipzig, Germany. \\ Email: diyar.saeed@med.uni-duesseldorf.de.
}

Submitted Sep 24, 2018. Accepted for publication Oct 10, 2018.

doi: $10.21037 /$ acs.2018.10.02

View this article at: http://dx.doi.org/10.21037/acs.2018.10.02

\section{Clinical vignette}

Selected patients who fail to be weaned from veno-arterial extracorporeal membrane oxygenation (VA-ECMO) may be considered for durable left ventricular assist devices (LVADs). These high-risk patients are usually converted to a cardiopulmonary bypass (CPB) machine at the time of VAD implantation surgery. The CPB may cause activation of the systemic inflammatory response with associated deleterious effects on the coagulation system that can lead to bleeding, thrombosis and increased blood product transfusion (1). In a recent study from our group, we were able to demonstrate the advantages of omitting CPB at the time of VAD implantation surgery $(2,3)$. Based on the outcome of our study, LVAD implantation for patients on VA-ECMO is performed at our institution without conversion to $\mathrm{CPB}$. We describe here the technique of HeartMate 3 (Abbott, Chicago, IL, USA) implantation in a patient on VA-ECMO.

The case is a 62 -year-old male patient with dilative cardiomyopathy. The patient was listed high-urgent for heart transplantation. However, the clinical condition of the patient deteriorated, necessitating peripheral VA-ECMO implantation on an emergency basis. As weaning from VAECMO was not feasible and due to patient's characteristics (body mass index and blood group), a long waiting time for heart transplantation was expected, we decided to proceed with HeartMate 3 implantation after five days of VAECMO support. The patent was neurologically intact but on renal dialysis at the time of LVAD implantation.

\section{Surgical technique}

\section{Preparation}

The pump implantation is performed using a traditional sternotomy approach. A minimally invasive approach may alternatively be used. Intravenous heparin is administrated with target activated clotting time (ACT) of $>400$ s. Cell Saver (LivaNova PLC, London, UK) is used during the procedure.

\section{Exposition}

The first step is to adequately luxate the heart using the socalled "deep stich", which we traditionally use at time of offpump bypass surgery. The deep stich should be placed just above the inferior vena cava to avoid worsening of mitral valve insufficiency and subsequent lung edema (video).

\section{Operation}

The next step is to fix the metal ring of the pump after identifying the cannulation site. Four to five nonresorbable pledgeted Ethibond sutures are used, followed by a running 2-0 Prolene suture (video). It is crucial to maintain adequate haemostasis to avoid postoperative bleeding. Both HeartMate 3 and HeartWare HVAD (Medtronic, Minneapolis, MN) may be used. In this 
video, HeartMate 3 pump is implanted. This step is followed by the most critical step of the procedure, which is coring the left ventricular apex and pump insertion. We always perform the implantation on a beating heart. Alternatively, either ventricular fibrillation, rapid pacing (170 beats/min), or intravenous adenosine $(25 \mathrm{mg}$ ) can be used to create the apical access in the middle of the metal ring. It is important to de-air the system at this point, while the cardiac apex is still luxated. We prefer using a HeartWare coring knife for the procedure, because the current HeartMate 3 coring knife may cause more blood loss. However, the new HeartMate 3 surgical tools may also facilitate coring of the left ventricle with minimal blood loss. Afterwards, tunnelling of the drive line is performed in the usual manner. The final step is performing the outflow anastomosis to the ascending aorta after the application of a side clamp (video). This step is followed by adequate de-airing of the pump through an outflow graft and aorta and confirming adequate positioning of the inflow cannula under echocardiography guidance.

\section{Completion}

The VA-ECMO is then gradually weaned and the pump is simultaneously started, accompanied by inotropic support for the right ventricle. After effective haemostasis, the chest wall is closed. The VA-ECMO cannulas are then removed. In patients with severe right ventricular failure, a right VAD (RVAD) may be also implanted under VA-ECMO support.

This patient was extubated after 3 days and kidney function recovered. Apart from repeated gastrointestinal bleeding, the postoperative course was uneventful and the patient is now awaiting heart transplantation.

\section{Comments}

\section{Clinical results}

Starting from January 2010, over 75 patients with prior VA-ECMO were supported with permanent VAD at our institution. Thirty-five patients with mean age of $54 \pm 12$ years underwent LVAD implantation (HeartWare HVAD $=27$ and HeartMate $3=8$ ) on VA-ECMO without using CPB after a mean VA-ECMO support duration of $7 \pm 6$ days. The outcome of LVAD candidates with prior VAECMO remains poor, with higher rate of postoperative right ventricular failure and 1-year survival of merely $55 \%$ (4). Therefore, many centers turn patients down for permanent
VAD implantation once VA-ECMO is implanted. In our opinion, patient selection is a key determining factor for an optimal postoperative outcome particularly, in these patients. Therefore, a multicenter VA-ECMO-VAD registry was initiated in 2017. More than ten large volume centers are currently involved in the registry with a total of $>450$ patients. The results will be presented and published in near future. Preliminary data analysis shows that the model of end stage renal disease (MELD) score on VAECMO is the most important parameter determining outcomes in these patients.

\section{Advantages}

There is a tremendous difference in the characteristics and functional status of patients undergoing VAD implantation after VA-ECMO support (3). These patients are sicker, the majority of them have a history of cardiopulmonary reanimation, are on ventilator support prior to VAD surgery, have so-called acute lung injury on VA-ECMO with respiratory dysfunction, have a higher rate of right ventricular dysfunction and, most importantly, a higher bleeding tendency. Based on previous studies from our institution (3), we were able to show that implanting a VAD system in a patient with VA-ECMO is feasible. The amount of blood loss is less and a lower total number of blood transfusions were necessary when no $\mathrm{CPB}$ circuit was used.

\section{Caveats}

The main disadvantage of this technique is the difficulty of direct inspection of the left ventricle. Therefore, conversion to $\mathrm{CPB}$ is mandatory in patients with evidence of a left ventricle thrombus. Conversion to $\mathrm{CPB}$ is also mandatory for patients requiring concomitant procedures at the time of LVAD implantation, for instance, aortic valve replacement.

\section{Acknowledgements}

Some of the videos shown here were originally published in The Multimedia Manual for Cardio-Thoracic Surgery (MMCTS) at http://mmcts.org/tutorial/494.

\section{Footnote}

Conflicts of Interest: The authors have no conflicts of interest to declare. 


\section{References}

1. Welsh KJ, Nedelcu E, Bai Y, et al. How do we manage cardiopulmonary bypass coagulopathy? Transfusion 2014;54:2158-66.

2. Abdeen MS, Albert A, Maxhera B, et al. Implanting permanent left ventricular assist devices in patients on veno-arterial extracorporeal membrane oxygenation support: do we really need a cardiopulmonary bypass machine? Eur J Cardiothorac Surg 2016;50:542-7.

Cite this article as: Maxhera B, Sipahi NF, Albert A, Boeken $\mathrm{U}$, Lichtenberg A, Saeed D. Preoperative and intraoperative extracorporeal membrane oxygenation adoption for long-term left ventricular assist device implantation. Ann Cardiothorac Surg 2019;8(1):167-169. doi: 10.21037/acs.2018.10.02
3. Saeed D, Assmann A, Abdeen M, et al. Implanting permanent left ventricular assist devices in patients on veno-arterial extracorporeal membrane oxygenation support. Multimed Man Cardiothorac Surg 2016;2017. doi: 10.1510/mmcts.2016.003.

4. Maxhera B, Albert A, Ansari E, et al. Survival predictors in ventricular assist device patients with prior extracorporeal life support: selecting appropriate candidates. Artif Organs 2014;38:727-32. 